International Journal of Engineering \& Technology, 7 (4.33) (2018) $472-478$.
International Journal of Engineering \& Technology
WPC
Rebsite $:$ www.sciencepubco.com/index.php/IJET
Research paper

\title{
Drift Compensation for pH IsFET Sensor Using NARX Neural Networks
}

\author{
Mohammad Iqwhanus Syaffa Amir ${ }^{1 *}$, Md Rizal Othman', Mohd Ismahadi Syono² \\ ${ }^{1}$ Faculty of Electrical and Electronics Engineering, University Malaysia Pahang, Pekan Campus, 26600 Pekan, Pahang, Malaysia \\ ${ }^{2}$ Photonics R\&D, MIMOS Semiconductor (M) Sdn Bhd, Technology Park Malaysia, Kuala Lumpur, Malaysia \\ *Corresponding author E-mail: syaffa@gmail.com
}

\begin{abstract}
This paper introduces a Nonlinear Autoregressive Neural Network (NARX) to predict the sensor error of IsFET pH drift with accuracy over the long period. The Bayesian Regularization (BR) backpropagation was used as network training function for this problem and combined with different delay and hidden layer. The results were compared to predict the sensor error in buffer solution $\mathrm{pH} 4$, $\mathrm{pH} 7$ and pH 10 over the time. The NARX performance will be measure based on the value of Mean Squared Error (MSE) and coefficient of determination $\left(\mathrm{R}^{2}\right)$. The results proved by using Bayesian Regularization with 10 hidden nodes and 50 delays produced the accurate sensor error prediction. This research will provide the significant contributions to the implementation of IsFET $\mathrm{pH}$ sensor drift compensation over the time.
\end{abstract}

Keywords: Artificial Neural Network; Drift; Bayesian regularization; Ion sensitive field effect transistor; Nonlinear autoregressive.

\section{Introduction}

Ion Selective field effect transistor (IsFET) based sensor is commonly used from 1970 because of the advantages of low cost fabrication, small size and robustness. PH IsFET sensor currently has wide applications for instant in medical, environment sensing, agriculture and liquid waste management. In industrial system applications, IsFET sensor needs to be used continuously in liquid based solution and most of the IsFET sensor have an issue to maintain the good analogue output. The output of IsFET sensors drift over the time during a slow-moving variation in the IsFET voltage threshold and establishes from a transport phenomenon at the interface between the solution and the insulator [1]. Each sensor will produce a unique drift characteristic due to the different effect factor, for instance, the type of $\mathrm{pH}$ solution, the substance of sensor surface and dimension. Therefore, the compensation approaches cannot depend on mathematical method and have to develop in any degree voltage variation of temporal gate. In [2] conducted study to develop a physical model for drift in $\mathrm{pH}$ ISFETs. They found that from the physical model of the sensor, it can reduce the drift effect and contribute a good output. In [3] an analytical method to overcome the drift of Ion-Selective Field Effect Transistors (ISFETs). He found that the theoretical basis for the proposed correction technique would minimize the drift effect issues. In [4] developed a smart IsFET sensor system with drift and temperature correction for continuous monitoring. This implementation focus on optimum biasing current for ISFET and time-variant compensation algorithm. In [5] propose an improvement of bridge-type floating source circuit read-out circuit technique. In [6] studied the diffusion sensor interface and sensor insulation development that is more specific to the sensor element improvement. The development protective structure of sensor element using semiconductor technologies was done by [7]. They claimed that their research capable in reducing the drift issues. Another research was done by [8] specific on the sensor development using Zirconium dioxide gated Ion Sensitive Field Effect Transistors (IsFETs). In [9] developed a low noise differential read-out circuit and an insulating layer for ISFET sensor. In [10] improved the interpretation of IsFET signals in mixed-ion environments. The improvement of the ISFET sensor readout board circuit using differential measurements correlated double sampling did by [11]. In [12] developed readout board source and drain follower circuit of IsFET sensor. To counter the drift issues in IsFET sensor, in [13] investigated the development of readout board current mode circuit. In 14] developed the differential measurement readout board. In [15] focused on the readout board improvement in reset switch on the IsFET gate. In [16] implemented by applying source voltage compensation of the IsFET sensor. In [17] proposed a new method using a time-domain correction technique for uncertainty in Sensors Based on FieldEffect Transistors (FETs). Lately in [18] have implemented a Machine Learning algorithm to compensate the temperature for Ion-Sensitive Field-Effect Transistor (ISFET) sensor.

The crucial challenge for drift correction has always lied in its unexpected behavior, and this issue is modeled as an exponential relationship of time. In order to overcome this, it is important to develop an algorithm that can learn the sensor characteristic drift parameters. Artificial Neural Network (ANN) algorithm is one of the most suitable application on the prediction of drift characteristic. The NARX architecture is a dynamic Artificial Neural Network that relates the modeled change based on lagged input-output parameters and error prediction [19]. The NARX Neural Network embedded memory provides a shorter way to generate the data information and back propagate the error 
of signal, which is reducing the prolonged dependencies. This type of Neural Network can contribute optimum predictions without losses of computational data in comparison with the traditional recurrent neural networks [20-21]. In time series prediction, NARX neural networks have been used [22] and gave a great achievement in solving most of the non-linear problem. Recent researches have presented the NARX model advantages in diverse fields [23-26]. The aim of this paper is to solve the nonlinear drift effect that exists in the IsFET reading values. Three similar types of IsFET sensors were used with three $\mathrm{pH}$ test buffer solutions that are $\mathrm{pH} 4, \mathrm{pH} 7$ and $\mathrm{pH} \mathrm{10.} \mathrm{The} \mathrm{neural} \mathrm{network} \mathrm{that} \mathrm{used} \mathrm{in} \mathrm{this} \mathrm{paper} \mathrm{is} \mathrm{the}$ nonlinear autoregressive exogenous model. The experiment output of the nonlinear drift effect was done by using the MATLAB simulation.

\section{NARX model architecture}

Artificial Neural Networks (ANN) has been developed with the concept of a human brain, emulating the system of central nervous in the brain with a capability to establish a large-scale interconnected parallel network, and training of such networks to resolve particular issues or problems. ANN is a popular technique to solve nonlinear systems problem and the NARX Neural Network is one type of ANN family. The NARX advantage is with the same structure can be used in others problem and will minimize the design activity. The model of NARX able to do a comparison using the feedforward network with data from exogenous input. The method can reduce the variables required in fine-tune the network efficient and accurate compare to other ANN models. The NARX model definition known by

$u(t)=f\left(u(t-1), u(t-2), \ldots, u\left(t-n_{u}\right), k(t-1), k(t-2), \ldots, k\left(t-n_{k}\right)\right)$

show $u(t)$ and $k(t)$ stand for the outputs and inputs relatively for the discrete time step $(t)$ model. The variables for $n_{u}$ and $n_{k}$ are the output and input layers for the network. The function, $f$ is a nonlinear function and can be perform by the conventional feedforward network. The figure 1 show the function, $f$, used in this research.

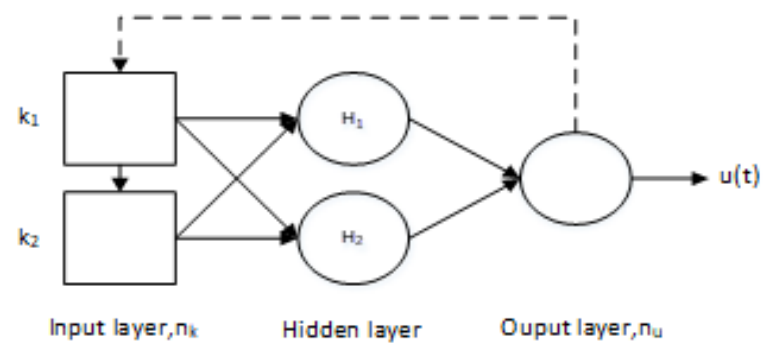

Figure. 1: NARX neural network with $\mathrm{n}_{\mathrm{k}}=\mathrm{n}_{\mathrm{u}}=2$ and $\mathrm{H}=2$

The two input configuration was used for NARX model, which perform the exogenous variable's data and feed-forward to the time delays. This model will produce single output of time prediction. Throughout the NARX model training, the actual data was used as input for the configuration of series-parallel network. After the training, the compute output was feedback to the model and acquired the calculation for the following prediction process. The optimization of this model is by decreasing the training iteration value. Normally, training involved specific hidden nodes, layer and delay value to establish the neural network connections. In this research, the value for weight and bias will be start with random number and will be change within the iteration process of developing the network model.

\section{Experimental procedure}

Experiment was conducted by using three wireless IsFET sensors for instant IsFET 1, IsFET 2 and IsFET 3 to measure $\mathrm{pH} 4, \mathrm{pH} 7$ and $\mathrm{pH} 10$ solutions. All sensors were setup in the shield box with no interference of lights and any frequency. This is important to avoid any disturbance that might affect the reading of the sensors. The temperature of the room was set 25 Celsius \pm 1 Celsius and the wireless sensors were set in an auto read mode for every 30 minutes and was automatically sent signal to the receiver. All data collections for each sensor and $\mathrm{pH}$ was collected for a period of 1 month. The data had been saved in the LabVIEW machine for each $\mathrm{pH}$ solution and the results can be seen in Figure 2.

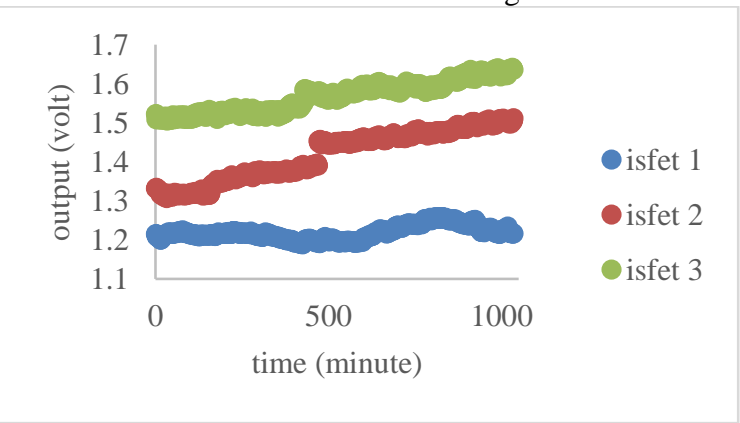

(a)

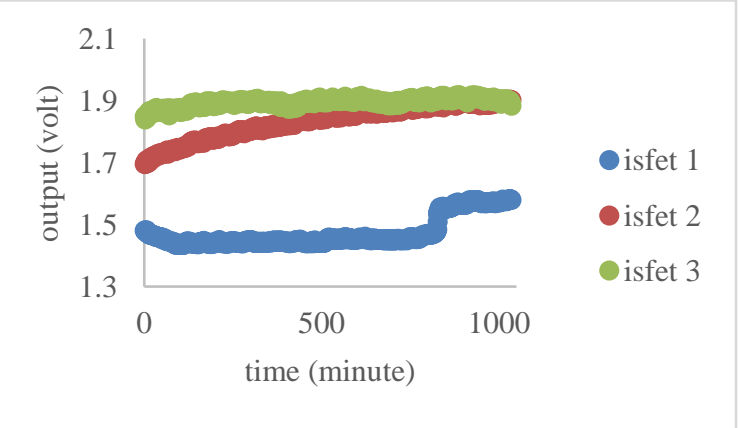

(b)

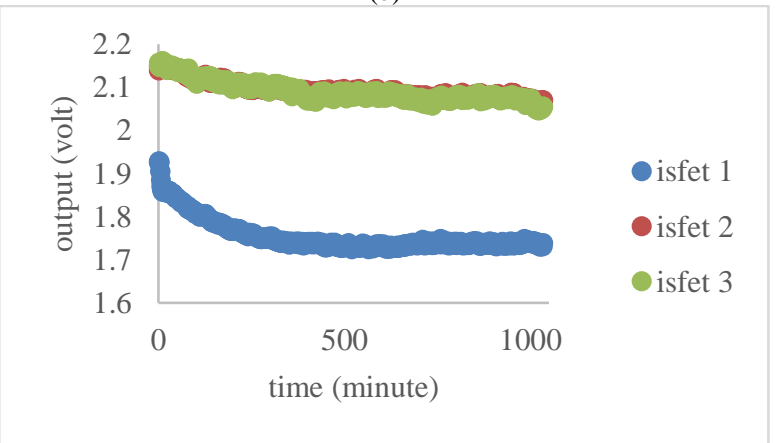

(c)

Fig. 2: Data collected for (a) $\mathrm{pH} 4$, (b) $\mathrm{pH} 7$ and (c) $\mathrm{pH} 10$ in one month

From the graphs, it can be seen clearly that the output voltage keeps on changing without any $\mathrm{pH}$ change. This drift situation needs to be fixed in all sensors used. The collected data was used to create models by using NARX neural networks and the models were generated by matching a sensor and all possible combinations of other sensors. The model was tested against the data to find the correlations.

\section{Results and discussion}

Our experimentation has focused on the compensation of drift $\mathrm{pH}$ IsFET sensor using nonlinear autoregressive recurrent neural network (NARX) for three different types of solutions namely $\mathrm{pH}$ 4, $\mathrm{pH} 7$ and $\mathrm{pH} 10$. In this section, the best achieved results for using the NARX ANN model in predicting and correcting the $\mathrm{pH}$ IsFET 
sensor are presented. The NARX neural network that built in this paper used 50 time delays and 10 hidden nodes. The input layer for this neural network is the sensor voltage and the sensor error value is the output layer. The neural network performance has been calculated form the value of mean squared error (MSE). The hyperbolic tangent sigmoid transfer function was used as hidden layer activation function and the output layer was in linear function. The $70 \%$ of sensor data were used for training, $15 \%$ for validation and another $15 \%$ for testing. All the processes for the NARX model training and testing were executed using MATLAB software.

Table 1 shows the comparison of coefficient determination or $\mathrm{R}^{2}$ for different predictive algorithms that are Bayesian Regularization (BR), Levenberg-Marquardt (LM) and Scaled Conjugate Gradient (SCG). After five simulations, the average regression recorded in BR is the best $(0.999248 \approx 1)$ compared to Levenberg-Marquardt and Scaled Conjugate Gradient. This shows that the Bayesian Regularization training algorithm has better performance among others in predicting the sensor error of the IsFET sensors.

Table 1: Comparison of $\mathrm{R}^{2}$ for different model performance

\begin{tabular}{|c|c|c|c|}
\hline \multirow{2}{*}{ Simulation } & \multicolumn{3}{|c|}{ Regression, $\mathrm{R}^{2}$} \\
\cline { 2 - 4 } & $\mathrm{BR}$ & $\mathrm{LM}$ & $\mathrm{SCG}$ \\
\hline 1 & 0.99926 & 0.99943 & 0.99879 \\
\hline 2 & 0.99926 & 0.9997 & 0.99901 \\
\hline 3 & 0.9992 & 0.99842 & 0.99877 \\
\hline 4 & 0.99926 & 0.99987 & 0.99901 \\
\hline 5 & 0.99926 & 0.99946 & 0.99772 \\
\hline Average & 0.999248 & 0.999186 & 0.99866 \\
\hline
\end{tabular}

The Mean Square Error (MSE) and the number of iteration (I) with different training algorithms are outlined in Table 2. The diversities between training processes were more noticeable as the number of delays enlarged. From this table, it can be noticed that the number of iterations in Levenberg-Marquardt are less than with Bayesian Regularization and Scaled Conjugate Gradient. This explains the Levenberg-Marquardt has a fast convergence in network training but the Bayesian Regularization much better in prediction capabilities compare to other algorithms. The MSE values recorded in Scaled Conjugate Gradient are high in every number of delays and that suggest Scaled Conjugate Gradient is not an effective algorithm in predicting the error of the IsFET sensors. Therefore, the implementation of Bayesian Regularization for this research gives the best performance with higher predictive capabilities as recorder in this table.

Table 2: Iterations (I) and Mean Square Error (MSE) for the model performance evaluation

\begin{tabular}{|c|c|c|c|c|c|c|}
\hline \multirow{2}{*}{ Delays } & \multicolumn{2}{|c|}{ BR } & \multicolumn{2}{c|}{ LM } & \multicolumn{2}{c|}{ SCG } \\
\cline { 2 - 7 } & MSE & I & MSE & I & MSE & I \\
\hline 75 & $4.695 \times 10^{-5}$ & 819 & $\begin{array}{c}4.963 \times 10^{-} \\
5\end{array}$ & 13 & $\begin{array}{c}6.256 \times 10^{-} \\
5\end{array}$ & 263 \\
\hline 50 & $4.600 \times 10^{-5}$ & 585 & $\begin{array}{c}4.917 \times 10^{-} \\
5\end{array}$ & 15 & $\begin{array}{c}5.812 \times 10^{-} \\
5\end{array}$ & 385 \\
\hline 25 & $4.681 \times 10^{-5}$ & 1000 & $\begin{array}{c}4.892 \times 10^{-} \\
5\end{array}$ & 14 & $\begin{array}{c}9.438 \times 10^{-} \\
5\end{array}$ & 84 \\
\hline 10 & $4.735 \times 10^{-5}$ & 490 & $\begin{array}{c}4.849 \times 10^{-} \\
5\end{array}$ & 16 & $\begin{array}{c}6.088 \times 10^{-} \\
5\end{array}$ & 136 \\
\hline 2 & $4.861 \times 10^{-5}$ & 940 & $\begin{array}{c}4.865 \times 10^{-} \\
5\end{array}$ & 29 & $\begin{array}{c}5.367 \times 10^{-} \\
5\end{array}$ & 237 \\
\hline
\end{tabular}

Figure 3-5 show graphical representation of $\mathrm{R}^{2}$ for sensor IsFET1, IsFET2 and IsFET3 in three different $\mathrm{pH}$ solutions that are $\mathrm{pH} 4$, pH7 and pH10 respectively. From all the graphs, it can be seen clearly that the differences between the data and the fitted regression line are small. Scrutinizing on the coefficient of determinations or $\mathrm{R}^{2}$ values recorded in each cases, the range of the $\mathrm{R}^{2}$ is between 0.95-0.99 which gives a good measure on how close the date is to the fitted regression line. This indicates that the NARX model fits well the data collected from all the IsFET sensors.

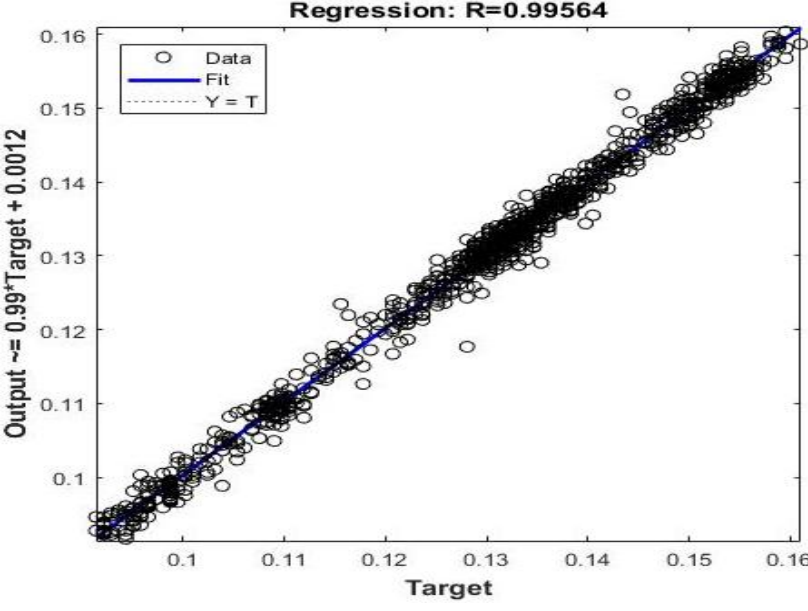

(a)

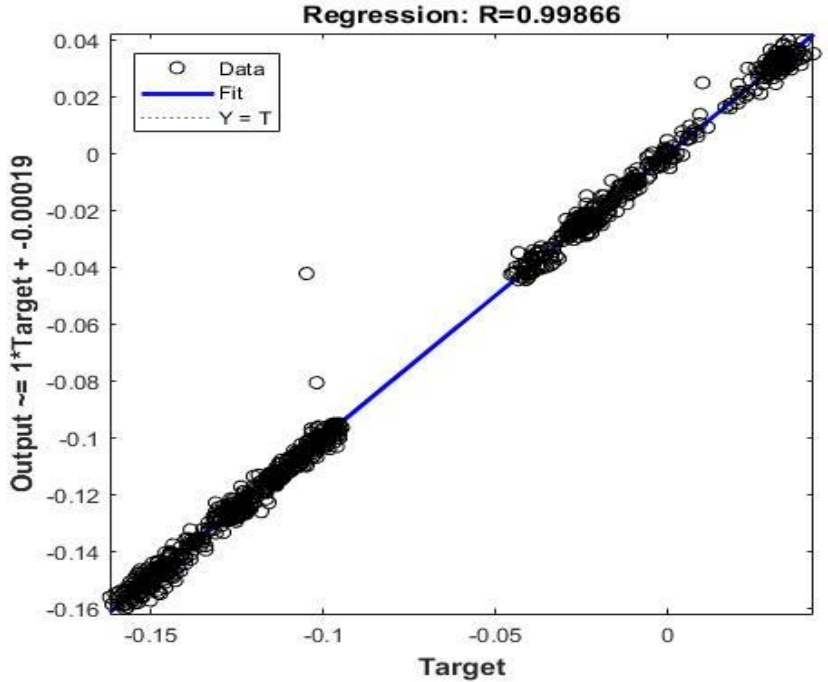

(b)

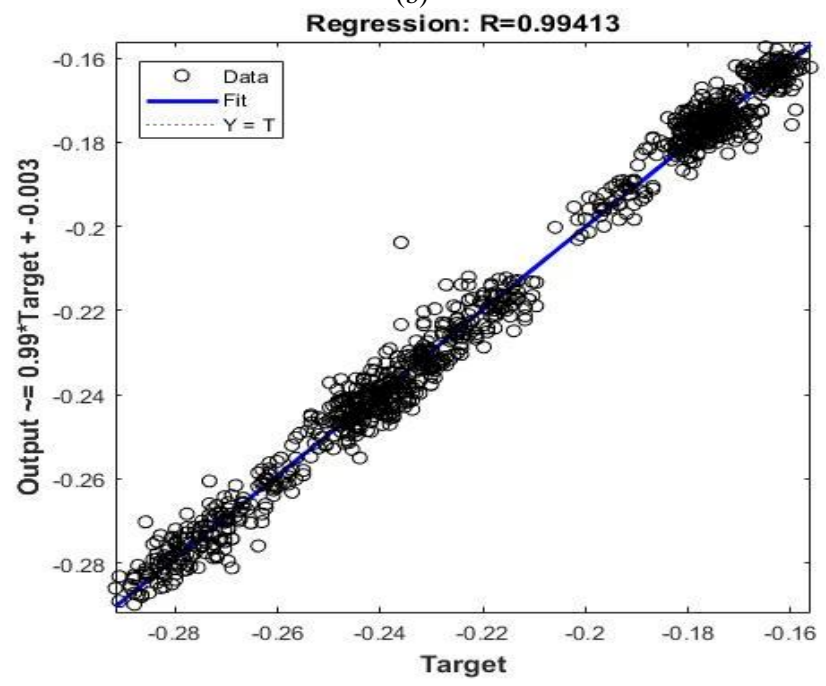

(c)

Fig. 3: Regressions of pH4 for (a) IsFET1, (b) IsFET2, (c) IsFET3 


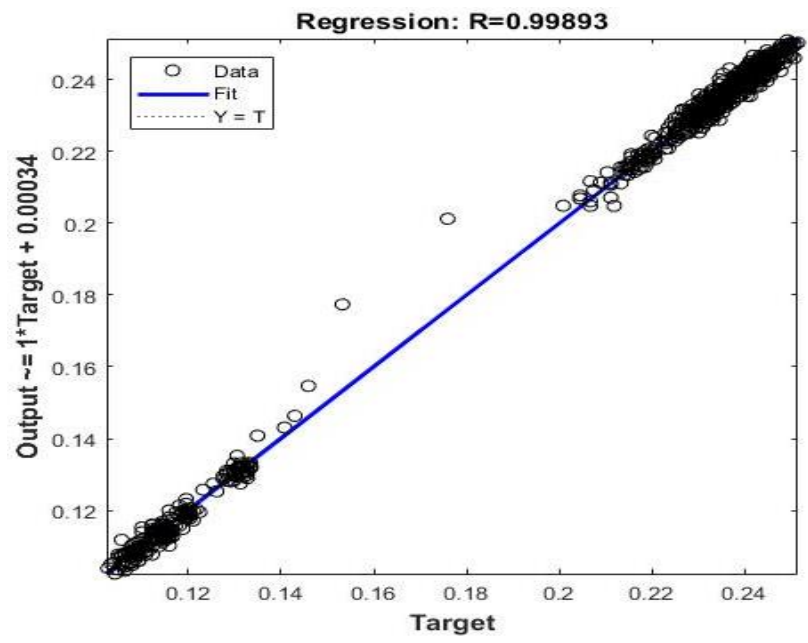

(a)

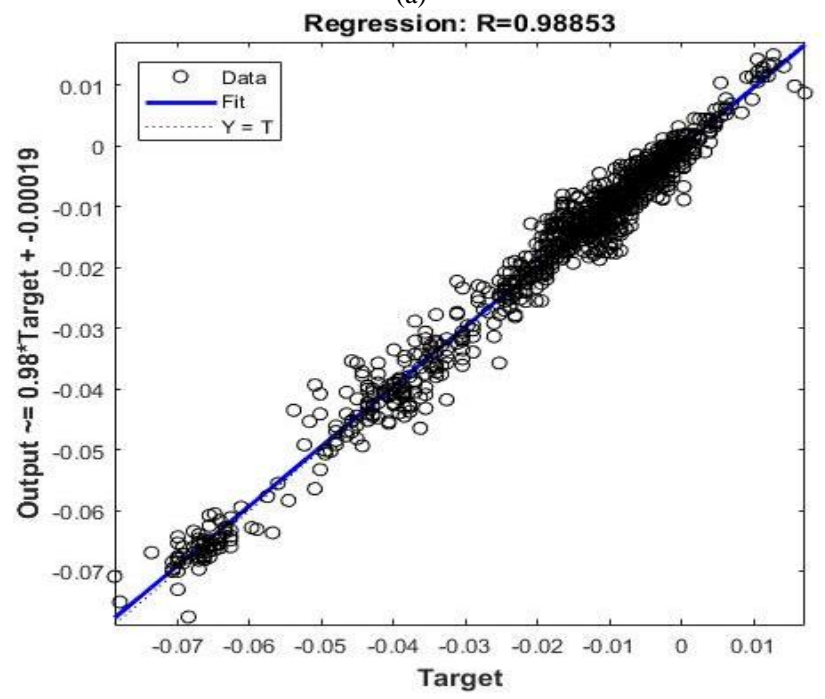

(b)

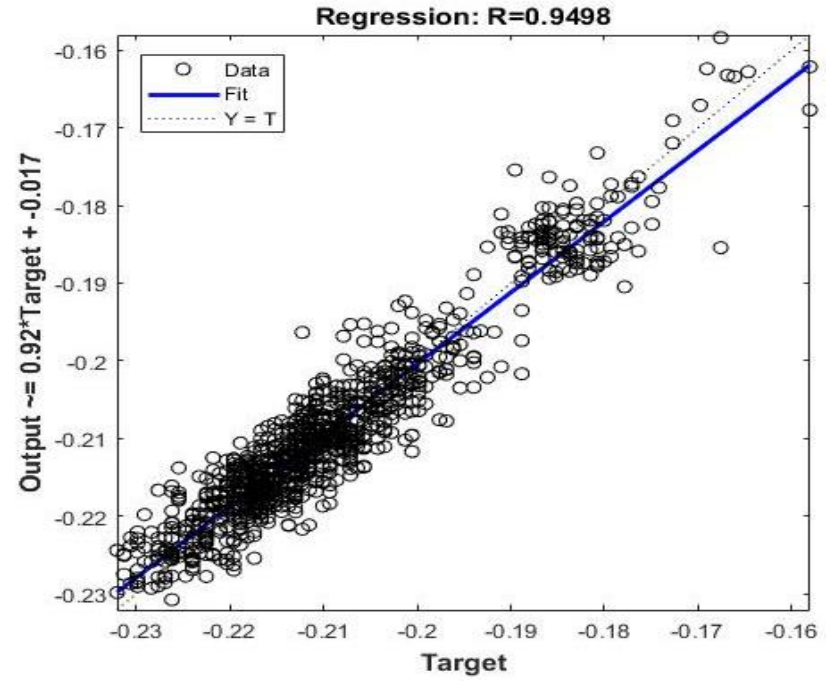

(c)

Fig. 4: Regressions of pH7 for (a) IsFET1, (b) IsFET2, (c) IsFET3

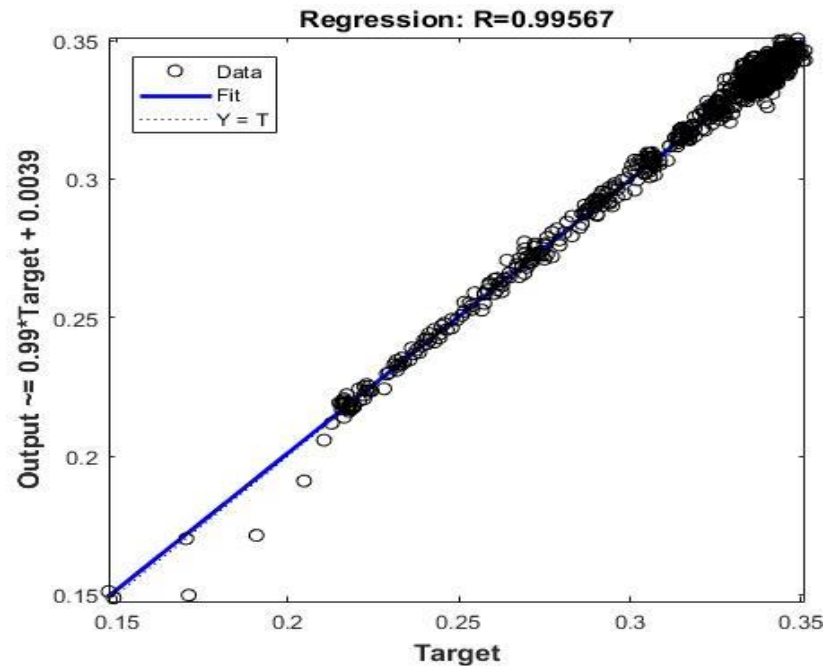

(a)

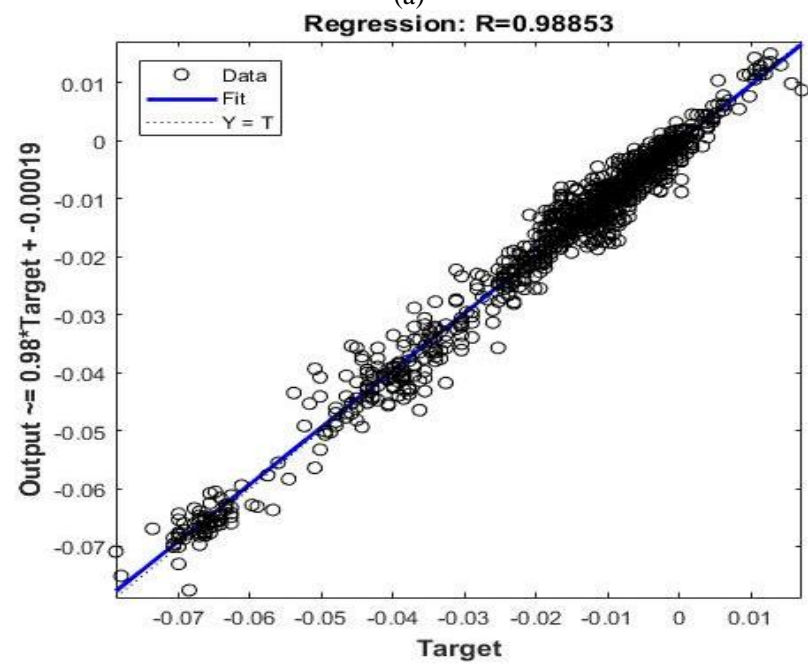

(b)

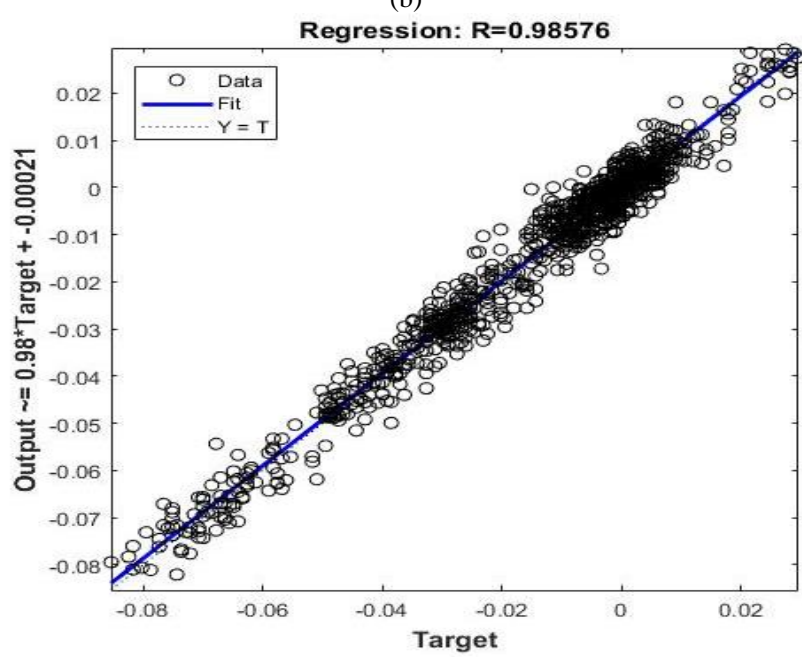

(c)

Fig. 5: Regressions of pH10 for (a) IsFET1, (b) IsFET2 and (c) IsFET3

Figure 6-8 show the original drifted data and the corrected data after implementing the NARX model with ten hidden nodes and fifty time delays in $\mathrm{pH} 4, \mathrm{pH} 7$ and $\mathrm{pH} 10$ solutions respectively. From the graphs, it can be seen clearly that the differences between measured and corrected values are seems to be large. This explained that the IsFET sensors behave unpredictably and drift issues consistently exhibit in an experiment. 


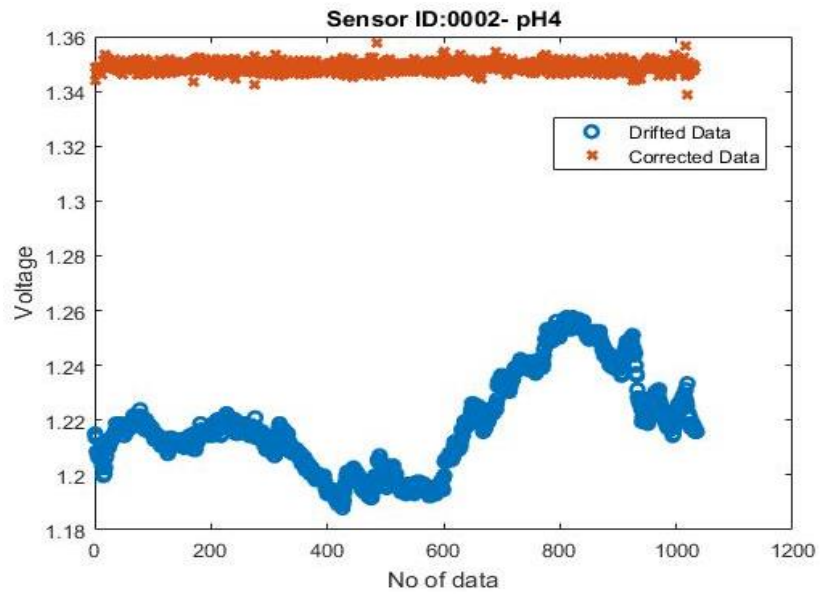

(a)

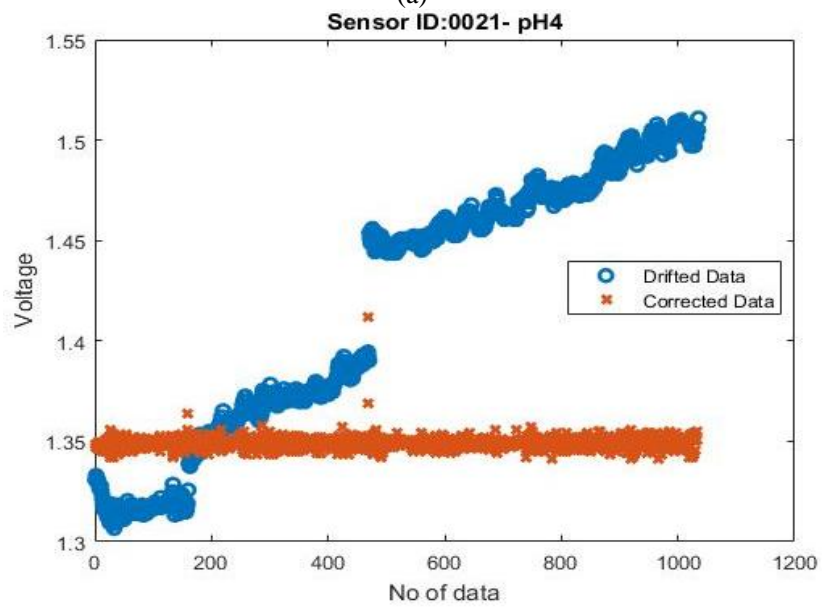

(b)

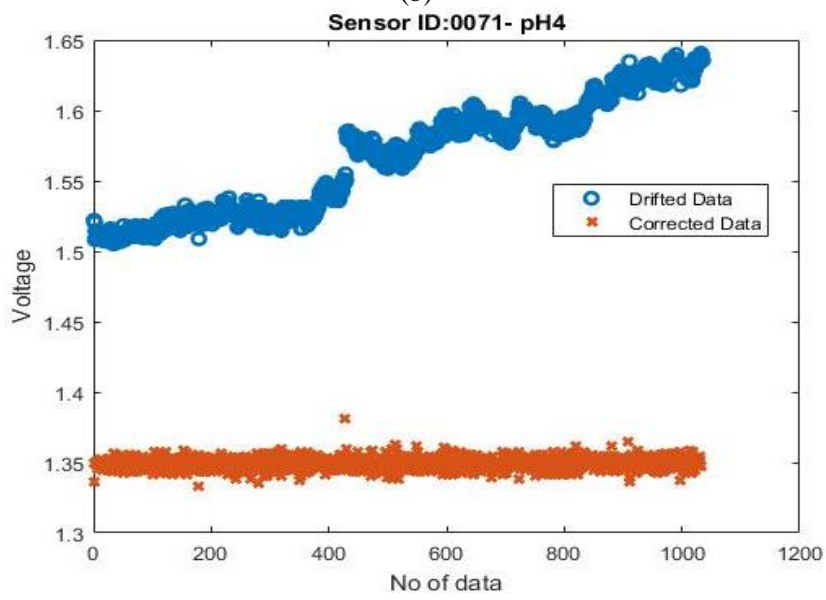

(c)

Fig. 6: Correction of pH4 for (a) IsFET 1, (b) IsFET 2, (c) IsFET 3

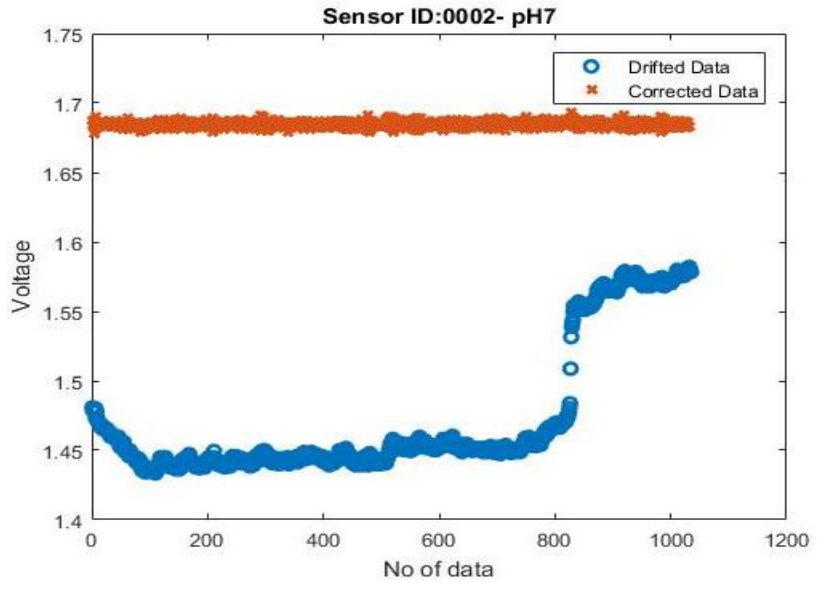

(a)

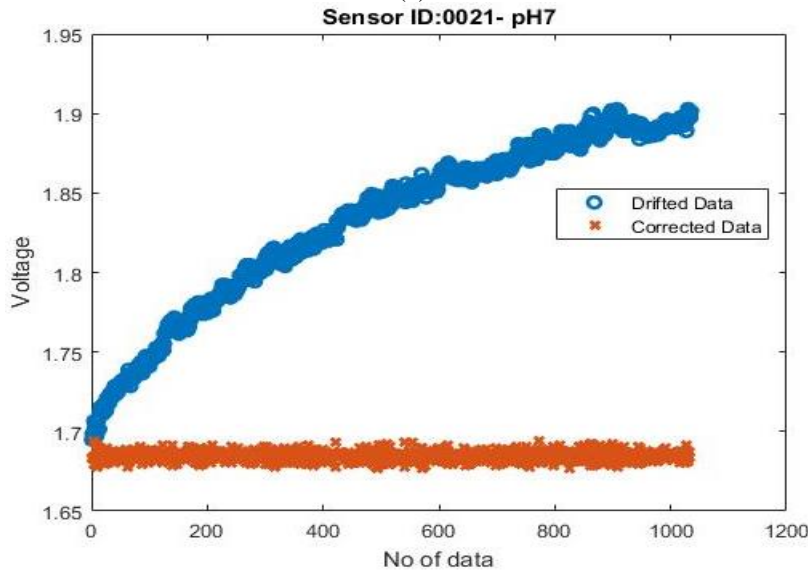

(b)

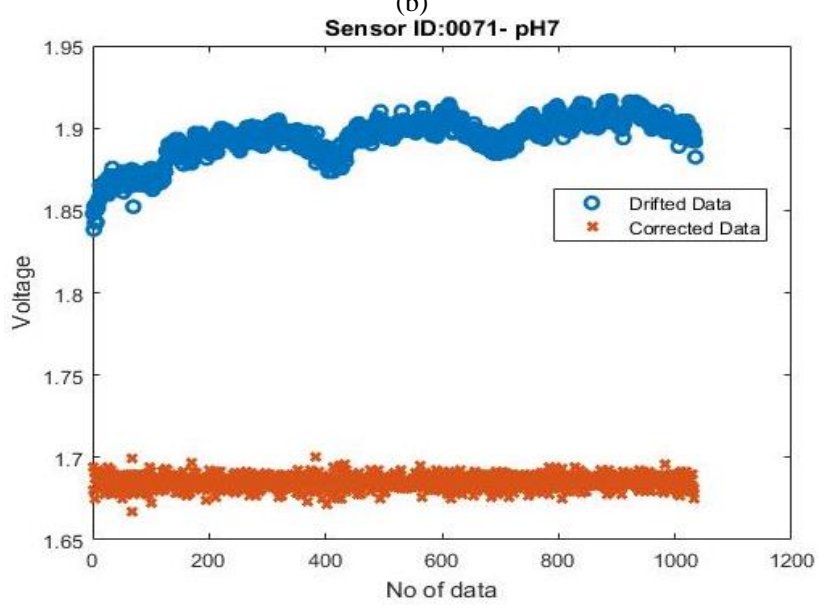

(c)

Fig. 7: Correction of pH7 for (a) IsFET 1, (b) IsFET 2, (c) IsFET 3 


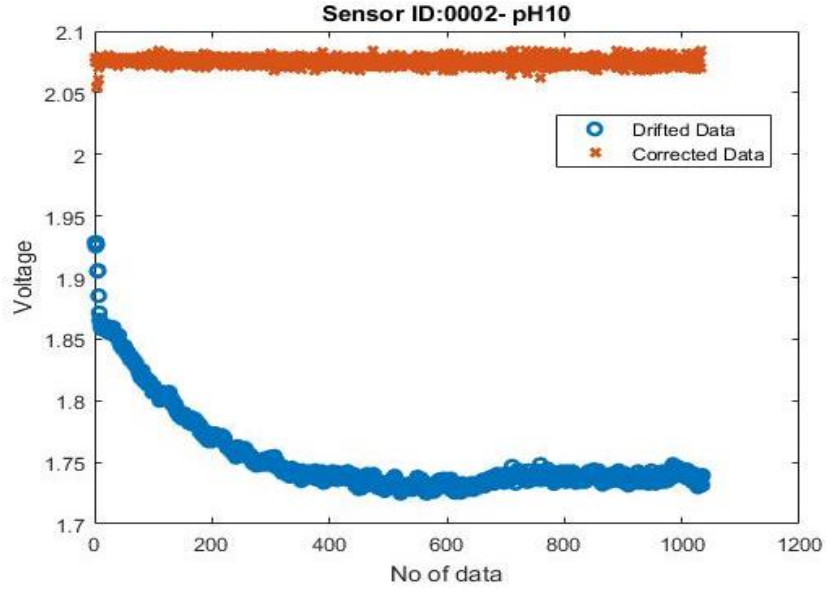

(a)

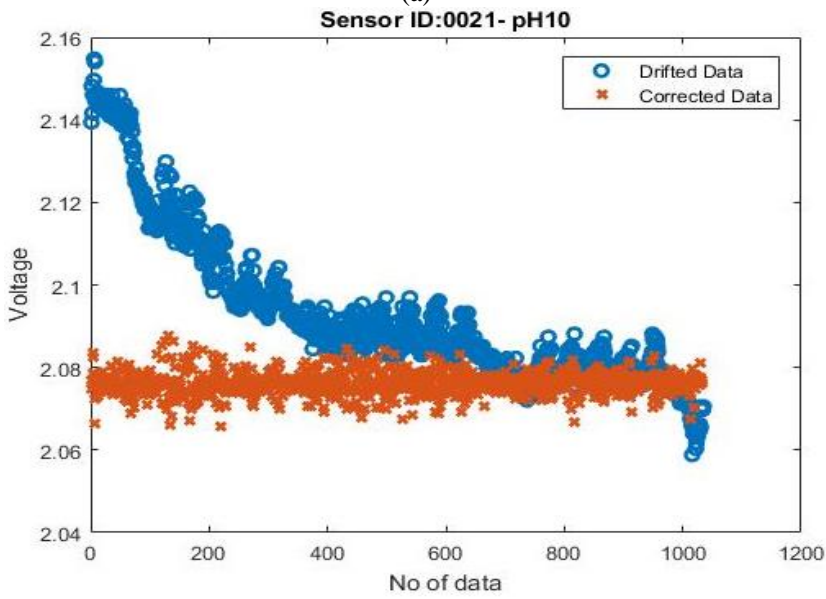

(b)

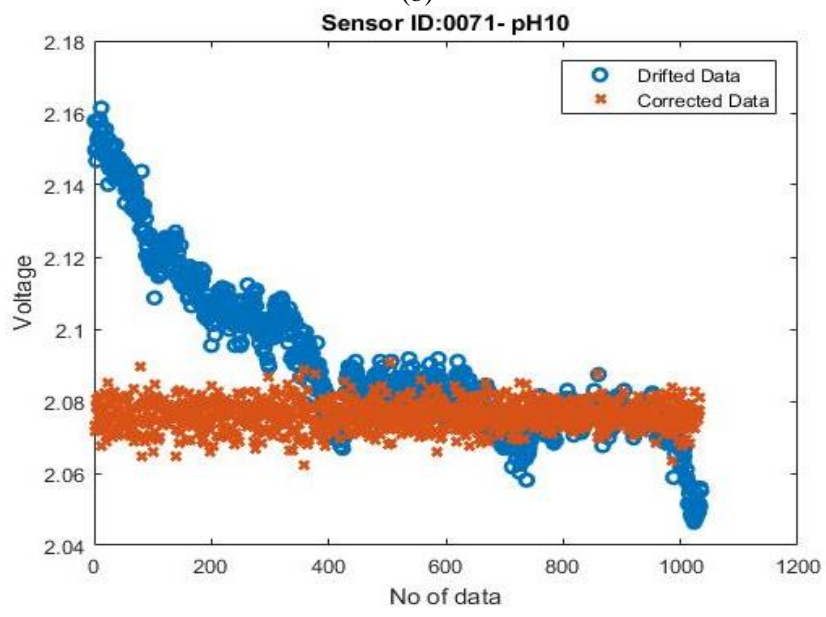

(c)

Fig. 8: Correction of pH10 for (a) IsFET 1, (b) IsFET 2, (c) IsFET 3

\section{Conclusion}

This paper showed the NARX neural network capabilities in sensor error prediction for $\mathrm{pH}$ buffer solution over the times. We acknowledged the precise and effective training algorithm using voltage data as input and predicted sensor error for a $\mathrm{pH} 4,7$ and 10 . Based on the MSE performance and training results, Bayesian Regularization with 10 hidden nodes and 50 delays was the precise model (MSE $=4.600 \times 10^{-5}, \mathrm{R}^{2}=0.99928$ ) for the sensor drift study. The outcomes acquired in this research proved the NARX model capability to predict sensor error over the long period. Nevertheless, this network depends on the training data availability, and the prediction relies on the input data quality applies during the training process. Further research will contain the compensation of
$\mathrm{pH}$ drift effect and the evaluation of the NARX implementation with the light and temperature effect's variables.

\section{Acknowledgement}

Authors gratefully thank Universiti Malaysia Pahang for the research grant (RDU1703231) and MIMOS Berhad for supporting this work by providing laboratory facilities during research.

\section{References}

[1] Moser, N., Lande, T. S., Toumazou, C., \& Georgiou, P. (2016) ISFETs in CMOS and emergent trends in instrumentation: A review. IEEE Sensors Journal, 16(17), 6496-6514.

[2] Jamasb, S., Collins, S. D., \& Smith, R. L. (1998). A physical model for threshold voltage instability in $\mathrm{Si} / \mathrm{sub} 3 / \mathrm{N} /$ sub 4/-gate H/sup+/sensitive FET's (pH ISFET's). IEEE Transactions on Electron Devices, 45(6), 1239-1245.

[3] Jamasb, S. (2004). An analytical technique for counteracting drift in ion-selective field effect transistors (ISFETs). IEEE Sensors Journal, 4(6), 795-801.

[4] Chen, D. Y., \& Chan, P. K. (2008). An intelligent ISFET sensory system with temperature and drift compensation for long-term monitoring. IEEE Sensors Journal, 8(12), 1948-1959.

[5] Chung, W. Y., Cruz, F. R. G., Yang, C. H., He, F. S., Liu, T. T., Pijanowska, D. G., Torbicz, W., Grabiec, P. B., \& Jarosewicz, B. (2010). CMOS readout circuit developments for ion sensitive field effect transistor based sensor applications. In J. W. Swart (Ed.), Solid State Circuits Technologies. London: IntechOpen, pp. 421-444.

[6] Sundaram, S., \& Sharma, N. N. (2010). Modeling interface diffusion as a mechanism for threshold voltage drift in $\mathrm{pH}$ sensors. Proceedings of the IEEE Sensors, pp. 2547-2550.

[7] Lee, S. K., \& Choi, S. Y. (2010). Improvement of drift characteristic to continuously measure $\mathrm{Al} 2 \mathrm{O} 3 \mathrm{pH}$-ISFET with the protective structure. Proceedings of the Meeting Abstracts, pp. 38-38.

[8] Chang, K. M., Chang, C. T., Chao, K. Y., \& Lin, C. H. (2010). A novel $\mathrm{pH}$-dependent drift improvement method for zirconium dioxide gated $\mathrm{pH}$-ion sensitive field effect transistors. Sensors, 10(5), 4643-4654.

[9] Jiao, L. H., \& Barakat, N. (2013). Ion-sensitive field effect transistor as a pH sensor. Journal of Nanoscience and Nanotechnology, 13(2), 1194-1198.

[10] Abdullah, W. F. H., Othman, M., Ali, M. A. M., \& Islam, M. S. (2010). Multiple feedforward classifiers by bagging for ion-sensitive field effect transistor sensor response. Proceedings of the IEEE International Conference on Computer Applications and Industrial Electronics, pp. 90-93.

[11] Das, M. P., \& Bhuyan, M. (2014). New ISFET interface circuits with noise reduction capability. Proceedings of the IEEE International Conference on Recent Advances and Innovations in Engineering, pp. 1-6.

[12] Uzzal, M. M., Zarkesh-Ha, P., Edwards, J. S., Coelho, E., \& Rawat, P. (2014). A highly sensitive ISFET using pH-to-current conversion for real-time DNA sequencing. Proceedings of the 27th IEEE International System-on-Chip Conference, pp. 410-414.

[13] Sohbati, M., \& Toumazou, C. (2014). A temperature insensitive continuous time $\Delta \mathrm{pH}$ to digital converter. Proceedings of the IEEE International Symposium on Circuits and Systems, pp. 37-40.

[14] Kalofonou, M., \& Toumazou, C. (2014). A low power sub- $\mu$ W chemical Gilbert cell for ISFET differential reaction monitoring. IEEE Transactions on Biomedical Circuits and Systems, 8(4), 565574.

[15] Hu, Y., \& Georgiou, P. (2014). A robust ISFET pH-measuring frontend for chemical reaction monitoring. IEEE Transactions on Biomedical Circuits and Systems, 8(2), 177-185.

[16] Moser, N., Lande, T. S., \& Georgiou, P. (2015). A novel pH-to-time ISFET pixel architecture with offset compensation. Proceedings of the IEEE International Symposium on Circuits and Systems, pp. 481484.

[17] Jamasb, S. (2016). A time-domain method for correction of instability in sensors based on field effect transistors (FETs). International Journal of Circuits, Systems and Signal Processing, 10, $119-125$. 
[18] Bhardwaj, R., Majumder, S., Ajmera, P. K., Sinha, S., Sharma, R., Mukhiya, R., \& Narang, P. (2017). Temperature compensation of ISFET based $\mathrm{pH}$ sensor using artificial neural networks. Proceedings of the IEEE Regional Symposium on Micro and Nanoelectronics, pp. $155-158$.

[19] Siegelmann, H. T., Horne, B. G., \& Giles, C. L. (1997) Computational capabilities of recurrent NARX neural networks. IEEE Transactions on Systems, Man, and Cybernetics, Part B (Cybernetics), 27(2), 208-215

[20] Lin, T. N., Giles, C. L., Horne, B. G., \& Kung, S. Y. (1997). A delay damage model selection algorithm for NARX neural networks. IEEE Transactions on Signal Processing, 45(11), 2719-2730.

[21] Diaconescu, E. (2008). The use of NARX neural networks to predict chaotic time series. WSEAS Transactions on Computer Research, 3(3), 182-191.

[22] Diaconescu, E. (2008). The use of NARX neural networks to predict chaotic time series. WSEAS Transactions on Computer Research, 3(3), 182-191.

[23] Al-Sbou, Y. A., \& Alawasa, K. M. (2017). Nonlinear autoregressive recurrent neural network model for solar radiation prediction. International Journal of Applied Engineering Research, 12(14), 4518-4527.

[24] Guzman, S. M., Paz, J. O., \& Tagert, M. L. M. (2017). The use of NARX neural networks to forecast daily groundwater levels. Water Resources Management, 31(5), 1591-1603.

[25] Pisoni, E., Farina, M., Carnevale, C., \& Piroddi, L. (2009). Forecasting peak air pollution levels using NARX models. Engineering Applications of Artificial Intelligence, 22(4-5), 593 602.

[26] Ruiz, L. G. B., Cuéllar, M. P., Calvo-Flores, M. D., \& Jiménez, M D. C. P. (2016). An application of non-linear autoregressive neural networks to predict energy consumption in public buildings. Energies, 9(9), 1-21. 